\title{
Endodomain Diversity in the Drosophila Dscam and Its Roles in Neuronal Morphogenesis
}

\author{
Hung-Hsiang Yu, ${ }^{1 *}$ Jacob S. Yang, ${ }^{1 *}$ Jian Wang, ${ }^{2}$ Yaling Huang, ${ }^{1}$ and Tzumin Lee ${ }^{1}$ \\ ${ }^{1}$ Department of Neurobiology, University of Massachusetts Medical School, Worcester, Massachusetts 01605, and ${ }^{2}$ Department of Entomology, University \\ of Maryland, College Park, Maryland 20742
}

\begin{abstract}
Drosophila Down syndrome cell adhesion molecule (Dscam) can be variably spliced to encode 152,064 distinct single-pass transmembrane proteins. In addition to 19,008 possible ectodomains and two alternative transmembrane segments, it may carry endodomains containing or lacking exons 19 and 23. Here, we determine the role of Dscam endodomain diversity in neural development. Dscam with full-length endodomain is largely restricted to embryogenesis. In contrast, most Dscams lack exons 19 and 23 at postembryonic stages. As implicated from the expression patterns, removal of Dscam exon 19-containing variants disrupts wiring of embryonic neurons while silencing of Dscam transcripts lacking exon 19 or exon 23 effectively blocks postembryonic neuronal morphogenesis. Furthermore, compared with exon 19-containing Dscam, transgenic Dscam without exon 19 is more efficiently targeted to neurites and more potently suppresses axon bifurcation in Dscam mutant neurons. In sum, Dscam with or without exon 19 in its endodomain is used to govern different stage-specific neuronal morphogenetic processes, possibly due to differences in protein targeting.
\end{abstract}

Key words: Dscam; endodomain; alternative splicing; miRNA silencing; neuronal morphogenesis; differential protein distribution

\section{Introduction}

One major challenge in the assembly of functional neural circuits is to distinguish self-branches from those of their neighboring neurons to prevent redundant connections. In Drosophila, Down syndrome cell adhesion molecule (Dscam) is primarily involved in this process during neuronal morphogenesis, including axonal guidance, axonal targeting and proper arborization of axons and dendrites (Wang et al., 2002; Chen et al., 2006; Zhu et al., 2006). Drosophila Dscam can encode 152,064 distinct Ig/fibronectintype-III cell adhesion molecules through alternative choices of 19,008 ectodomains, two transmembrane domains and 4 endodomains (Schmucker et al., 2000; Wang et al., 2004). The huge diversity in the ectodomain, which only binds to the same-kind ectodomain with high affinity, potentially underlies self-recognition for individual neurons (Wojtowicz et al., 2004). Repulsion as a consequence of the homophilic Dscam-Dscam interactions may mediate selfavoidance among the neurites derived from the same cell while permitting fasciculation of neurites of different origins, to ensure proper patterning of multiple growth cones migrating simultaneously (Matthews et al., 2007). Furthermore, elaboration of dendrites versus axons in the same neuron may involve different subsets of Dscam ectodomains, since a Dscam ectodomain can be selectively targeted

Received Dec. 2, 2008; revised Jan. 2, 2009; accepted Jan. 5, 2009.

This work was supported by National Institutes of Health and March of Dimes Birth Defects Foundation. We thank Dr. T. Kidd for sharing results on the genetic interaction between abl and Dscam before publication. We also thank Dr. B. Leung for critical reading of this manuscript and members of the Lee laboratory for helpful discussions through the entire project.

*H.-H.Y. and J.S.Y. contributed equally to this work.

Correspondence should be addressed to Tzumin Lee at the above address. E-mail: tzumin.lee@umassmed.edu. DOI:10.1523/JNEUROSC1.5743-08.2009

Copyright $\odot 2009$ Society for Neuroscience $\quad$ 0270-6474/09/291904-11\$15.00/0 to dendrites or axons depending on its transmembrane domain (Shi et al., 2007).

Compared with the well studied Dscam ectodomain and transmembrane domain, little is known about the role of Dscam endodomain in neuronal morphogenesis. Here, we knocked down specific Dscam endodomain isoforms to determine their roles in neuronal morphogenesis. A microRNA (miRNA)-based inducible RNA interference (RNAi) silences specific transcripts based on discrete 22-nucleotide sequences (Chen et al., 2007; Shi et al., 2007). This approach allows us to directly demonstrate the involvement of distinct isoforms at different places and times. It knocks down isoform expression after splicing, rather than at the transcriptional level through genomic manipulation, and should minimally affect the normal usage of isoforms. Using this technique, we are able to silence specific Dscam endodomain isoforms by targeting the exon 19- or exon 23-characteristic sequences, or the unique sequences around the junction between exon 18 and exon 20 or between exon 22 and exon 24 .

Elimination of Dscam isoforms either containing or lacking exons 19 and 23 revealed that the wiring of adult fly neural circuitry primarily involves Dscam isoforms lacking exon 19 and exon 23. However, Dscam with exon 19 plays a more important role than Dscam without exon 19 in supporting wiring of embryonic neural circuitry. In addition, Dscam variants with or without exon 23 are expressed in different compartments of the developing nervous system. Furthermore, transgenic Dscam without exon 19 is more efficiently targeted to neurites and more potently suppresses axon bifurcation in Dscam mutant neurons than exon 19-containing Dscam. This study demonstrates that Dscam governs diverse neural developmental processes not only through its variations in the extracellular and transmembrane domains, but also via the involvement of four different intracellular domains. 
Dscams with distinct endodomains mediate neural development at different stages and possibly in distinct contexts.

\section{Materials and Methods}

Transgenic constructs. Standard molecular biological techniques were used to generate UAS-19RNAi, UAS-23RNAi, UAS-18/20RNAi and UAS-22/24RNAi, which encode microRNAs (miRNAs) carrying unique Dscam sequences derived from exon 19, exon 23, junction between exon 18 and exon 20, and junction between exon 22 and exon 24, respectively. Their targets are indicated in Figure $1 A$, and the complete nucleotide sequences of the constructs are available upon request. UAS-18RNAi and four UAS-Dscam endodomain variants with the same ectodomain composition (UAS-Dscam [3.36.25.2]::GFPs) were described previously (Wang et al., 2004; Shi et al., 2007). Endogenous Dscam promoter-driven Dscam transgenic constructs were made by fusing $D$ scam [3.36.25.2]::GFP, which contain or lack exon 19, with the 4.5-kb Dscam promoter region (Wang et al., 2004).

Flies. Transgenic flies carrying various UAS-RNAis were obtained by $\mathrm{P}$ element-mediated germ line transformation with technical support from Genetic Services

Acute induction of UAS-transgenes by TARGET system. Larvae carrying two copies of tubulin promoter $(t u b P)-G A L 80^{t s}$ were cultured at the permissive temperature of $18^{\circ} \mathrm{C}$ throughout early development to repress GAL4-mediated transcription (McGuire et al., 2003). Wandering larvae were shifted to $38^{\circ} \mathrm{C}$ for $30 \mathrm{~min}$, followed by incubation at the nonpermissive temperature of $29^{\circ} \mathrm{C}$ for various periods.

Isolation and analysis of Dscam exon 15-24 cDNAs. Dscam exon 15-24 cDNAs were amplified by RT-PCR from mRNAs of embryos or the CNS of third-instar larvae, early pupae or adult flies with the primers CAGGTCTATGCCACAGGATTCA and GACTCTAGATTACACTGCCATAGTATC G. cDNAs were subcloned into pBluescript with HindIII and $\mathrm{Xba \textrm {I }}$ as the cloning sites. For each developmental stage, 40 independent subclones were individually analyzed for the presence or absence of exon 19 and/or exon 23 in their inserts. This was determined through analysis of exon-specific restriction enzyme sites and detection of isoformcharacteristic lengths of specific PCR products. The percentage of presence or absence of exon 19 or 23 among the analyzed clones is summarized in Figure 3G.

RNAi induction and phenotypic analysis. Targeted induction of UAS$R N A i$ involved use of various tissue-specific GAL4 drivers; and their phenotypic analysis mainly involved coexpression of various UASreporter genes. Rescue experiments by four different UAS-Dscam endodomain variants were analyzed in MARCM-labeled clones of $\alpha / \beta$ mushroom body (MB) neurons, which were induced $1 \mathrm{~d}$ before eclosion by heat shock for $30 \mathrm{~min}$ at $37^{\circ} \mathrm{C}$. Whole fly brains were prepared for immunostaining as described previously (Lee and Luo, 1999). A mouse monoclonal antibody (Dscam $18 \mathrm{mAb}$ ) raised against an epitope in exon 18 (ATLDKRRPDLRDELG) was used to detect all Dscam proteins (Shi et al., 2007), while a rabbit polyclonal antibody (Dscam 19 pAb) raised against an epitope in exon 19 (HSTWDPRRNPNLY) was used to detect exon 19-containing endogenous Dscam variants. These two Dscam antibodies could not work at the same time for double labeling, which might be due to close proximity of the epitopes bound by these antibodies. The anti-Dscam $18 \mathrm{mAb}$, anti-Dscam $19 \mathrm{pAb}, 1 \mathrm{D} 4 \mathrm{mAb}$, anti-GFP and anti-mCD8 mAb were used at 1:20, 1:1000, 1:50, 1:500 and 1:100, respectively. Fluorescence signals were captured with Zeiss confocal microscopy and processed using Adobe Photoshop.

\section{Results}

miRNA-based RNA interference permits differential silencing of Dscam transcripts encoding distinct endodomains

Based on the analysis of RT-PCR products, we have previously suggested that four possible Dscam endodomains, extending from exon 18 to exon 24, could be generated from independent alternative splicing of exon 19 and exon 23 (Wang et al., 2004). The full-length Dscam endodomain, located on the carboxyl side of the exon 17-encoded transmembrane/juxtamembrane segment, consists of 367 aa. It carries several putative SH3 binding sites, two 33 aa repeats that contain two consensus $\mathrm{SH} 2$ binding motifs, a proline-rich motif, a motif similar to the immunoreceptor tyrosine-based activation motif (ITAM-like), and a putative postsynaptic density, disc large and zo-I protein (PDZ)-binding motif (Schmucker et al., 2000; Brites et al., 2008). Skipping exon 19 produces a Dscam without a proline-rich motif (PPVP) and the ITAM-like $\left(\right.$ YEELx $_{(11)}$ YGHA) (Fig. $1 A$ ). Furthermore, due to use of a different splicing donor site, four additional amino acids (TVIS) are added at the junction where exon 18 connects with exon 20 (Fig. 1A). In contrast, omitting exon 23 makes a reading frame shift in exon 24 that results in the loss of the PDZ-binding motif (TMAV) (Fig. 1A).

The variations in the Dscam endodomain occur because of the presence or absence of exon 19 and exon 23 (Fig. 1A). To determine whether Dscam uses different endodomains in support of different neural developmental processes, we selectively knocked down Dscam transcripts containing or lacking exons 19 and/or exon 23 using a miRNA-based RNAi technology that permits silencing of specific transcripts based on presence of only 22 nucleotide-long unique sequences (Chen et al., 2007; Shi et al., 2007).

By targeting exon 19- or exon 23-characteristic sequences or the unique sequences at the exon-exon junctions derived from skipping of exon 19 or exon 23 (Fig. $1 A$ ), we successfully silenced the expression of specific Dscam transgenes based on the presence or absence of exon 19 or exon 23. Multiple independent transformants carrying UAS-19RNAi, UAS-18/20RNAi, UAS23RNAi, or UAS-22/24RNA $i$ were obtained and individually examined to identify the most effective RNAi transgenes for silencing their target-sequence-containing Dscam::GFPs in mature larval MB neurons (Fig. 1) (data not shown). GAL4-201Ydependent coinduction of distinct UAS-RNAis with a full-length Dscam (Dscam + 19+23::GFP) (Fig. $1 B-E$ ) or its derivative that skips both exon 19 and exon 23 (Dscam-19-23::GFP) (Fig. $1 \mathrm{~F}-I$ ) further revealed that UAS-19RNAi and UAS-23RNAi effectively antagonized the expression of Dscam +19+23::GFP (Fig. 1C,E) while minimally affecting the induction of Dscam-19-23::GFP which lacks the target sequences for these transgenic miRNAs (Fig. 1G,I). In contrast, UAS-18/20RNA $i$ and UAS-22/24RNAi effectively antagonized the expression of Dscam-19-23::GFP (Fig. 1F, $H$ ) while minimally affecting the induction of Dscam $+19+23::$ GFP (Fig. $1 B, D$ ). The specificity of $U A S-R N A$ is was further demonstrated by pan-neuronal induction of UAS-19RNAi and UAS-18/20RNAi to suppress the endogenous Dscam promoter-driven Dscam transgenes (supplemental Fig. S1 $A-F$, available at www.jneurosci.org as supplemental material). UAS-18/20RNAi silenced the expression of Dscam promoter-driven Dscam-19+23::GFP (supplemental Fig. S1E, available at www.jneurosci.org as supplemental material) while minimally affecting the induction of Dscam $+19+23:: G F P$ (supplemental Fig. $\mathrm{S} 1 B$, available at www.jneurosci.org as supplemental material). Conversely, UAS-19RNAi diminished the expression of Dscam promoter-driven Dscam+19+23::GFP (supplemental Fig. S1C, available at www.jneurosci.org as supplemental material) while hardly affecting the induction of Dscam-19+23::GFP (supplemental Fig. S1 F, available at www. jneurosci.org as supplemental material). These observations justified the use of UAS-19RNAi, UAS-18/20RNAi, UAS-23RNAi, and $U A S-22 / 24 R N A i$ for selectively silencing exon 19-containing $($ Dscam + 19), exon 19-lacking (Dscam-19), exon 23-containing $($ Dscam +23$)$ and exon 23-lacking (Dscam-23) Dscam transcripts, respectively. 


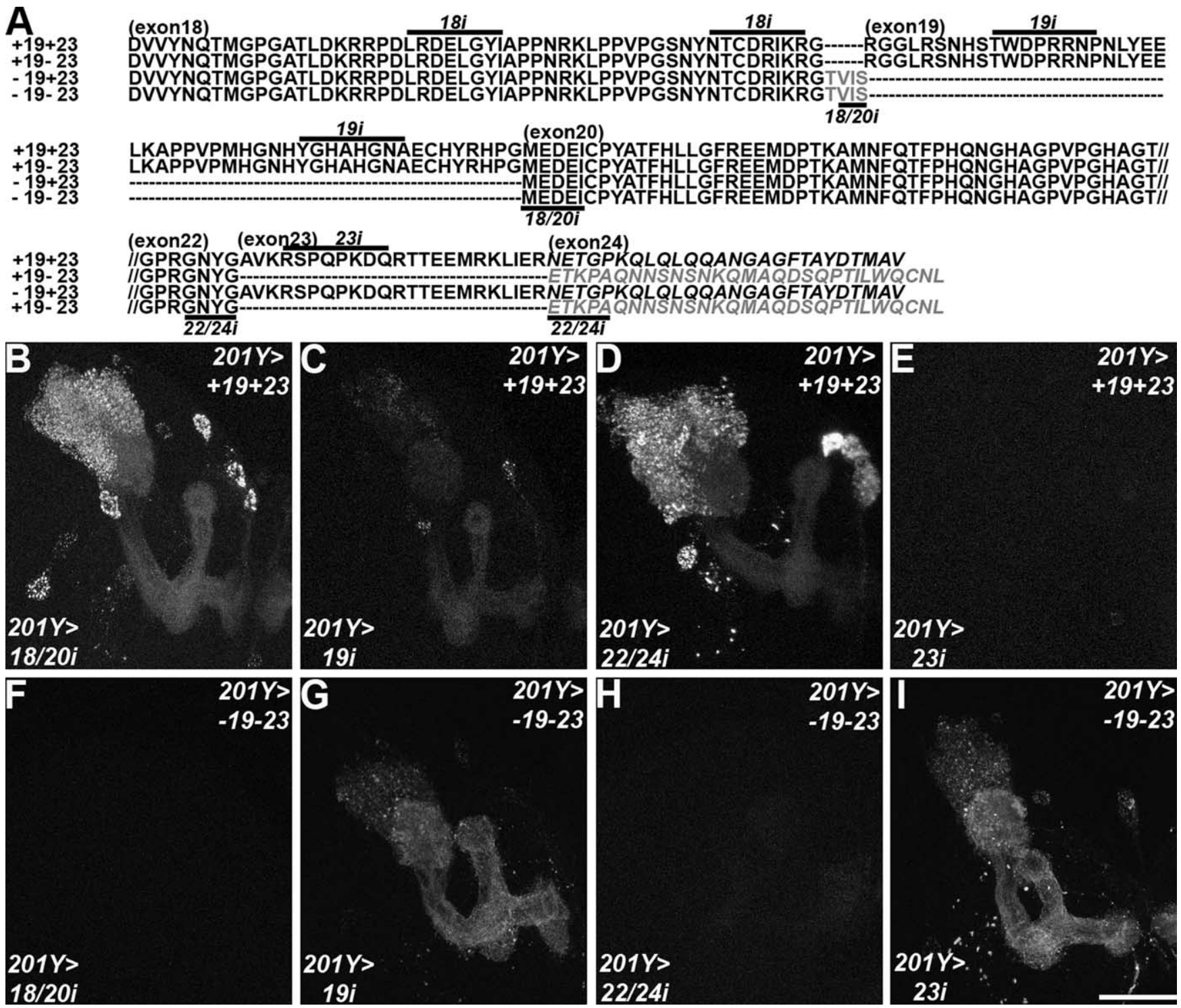

Figure 1. Amino acid sequences of four Dscam endodomain variants and silencing of various UAS-Dscam transgenes by specific UAS-RNAis. A, Amino acid sequences of four Dscam endodomain variants, full length $(+19+23)$, missing exon 19 only $(-19+23)$, lacking exon 23 only $(+19-23)$, and omitting both exon 19 and exon $23(-19-23)$, are aligned within the cytoplasmic domain from exon 18 to exon 24. Common sequence between exon 20 and exon 22 is not shown. Four extra amino acids (TVIS) are in the Dscam variants which skip exon 19 . Amino acids shown in italic result from a reading frame shift in exon 24 upon skipping of exon 23. Target sequences for different UAS-RNAis (18i, 18/20i, 19i, 22/24i and 23i) to eliminate different Dscam variants are indicated. $\boldsymbol{B}$-I, Composite confocal images of wandering larval MBs show suppression of the GAL4-201Y-dependent induction of UAS-Dscam + 19+23::GFP (B-E) and UAS-Dscam - 19-23::GFP $(\boldsymbol{F}-\boldsymbol{I})$ by UAS-18/20RNAi $(\boldsymbol{B}, \boldsymbol{F})$, UAS-19RNAi $(\boldsymbol{C}, \boldsymbol{G})$, UAS-22/24RNAi $(\boldsymbol{D}, \boldsymbol{H})$ and UAS-23RNAi $(\boldsymbol{E}, \boldsymbol{I})$, respectively. Scale bar (in this and all other figures), $50 \mu \mathrm{m}$.

Differential expression of Dscams with different endodomains

We have made a UAS-18RNAi transgene targeting the exon 18 shared by all Dscam transcripts, and previously demonstrated that ubiquitous induction of UAS-18RNAi abolished endogenous Dscam expression in the developing nervous system (Shi et al., 2007). To determine the effectiveness of our new RNAi transgenes in silencing the endogenous expression of Dscam, we examined how induction of individual or multiple Dscam RNAis affected the endogenous patterns of Dscam expression at different developmental stages.

In control embryos, immunostaining with an anti-Dscam exon 18 monoclonal antibody (Ab18) revealed that endogenous Dscam is concentrated on the ladder-like axonal tracts of the ventral ganglion (Fig. 2A). Such a stereotyped pattern of Dscam distribution was completely eliminated by tubulin promoter
(tubP)-GAL4-dependent ubiquitous induction of UAS-18RNAi (Fig. 2E), UAS-19RNAi plus UAS-18/20RNAi (Fig. 2D) or UAS23RNAi plus UAS-22/24RNAi (Fig. $2 H$ ). These results confirmed the efficacy of these RNAi reagents in silencing the expression of endogenous Dscam variants. It was noted that pan-neuronal induction of UAS-18RNAi by GAL4-C155 plus asense-GAL4 could not deplete the ladder-like axonal expression of endogenous Dscam, possibly due to late or insufficient induction of RNAi at the embryonic stage (data not shown). In contrast, residual Dscam persisted following tubP-GAL4-dependent induction of UAS-19RNAi, UAS-18/20RNAi, UAS-23RNAi or UAS-22/ 24RNA $i$ alone, even with multiple copies (data not shown). As to the induction of UAS-19RNAi (Fig. 2C) versus UAS-18/20RNAi (Fig. $2 B$ ), the ladder-like patterns of immunostaining, although weakened, looked morphologically indistinguishable from those seen in control embryos (Fig. $2 \mathrm{~A}$ ). In contrast, when endogenous 

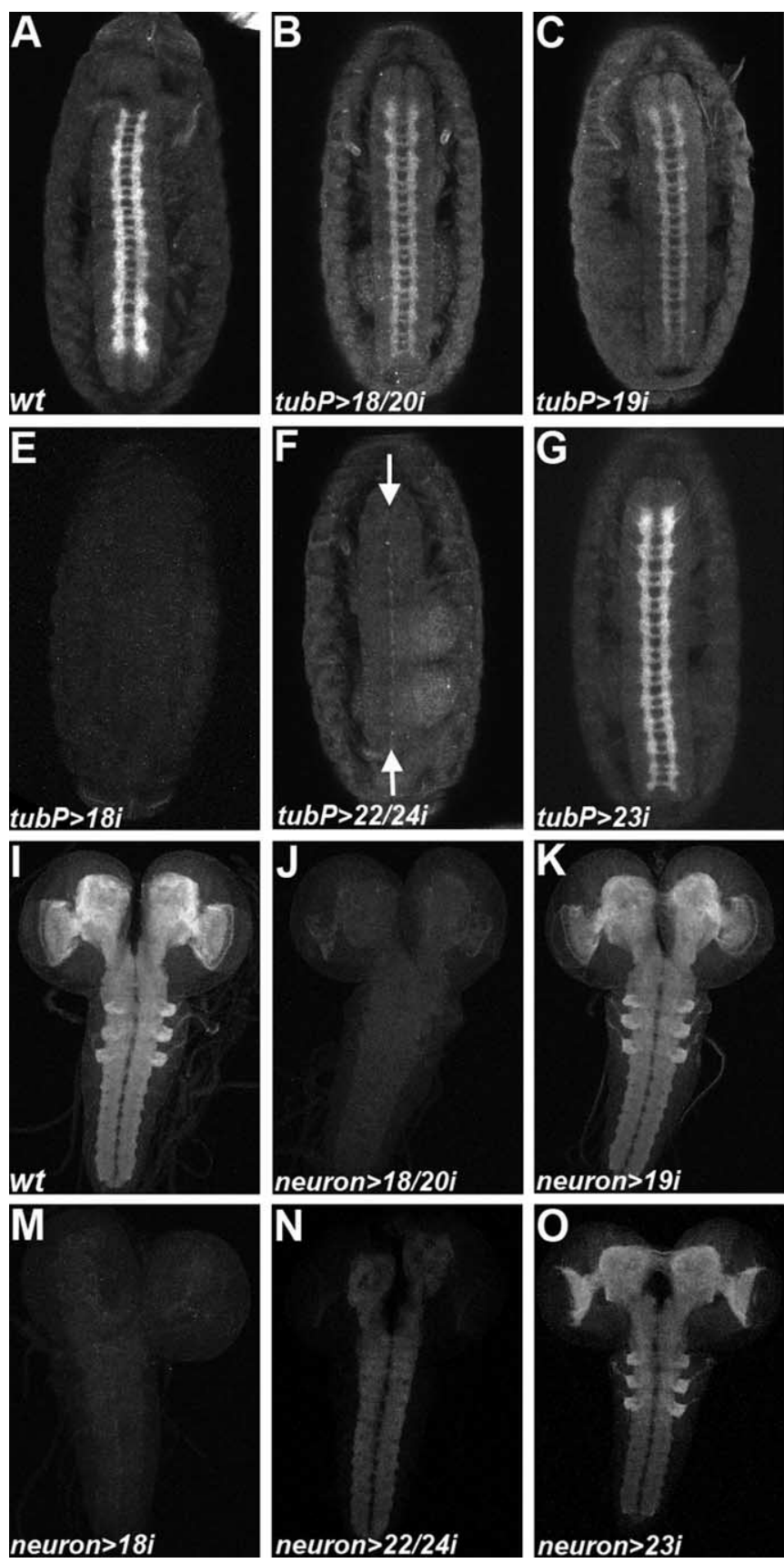

Figure 2. Elimination of specific Dscam endodoamin variants by transgenic miRNAs. $\boldsymbol{A}-\boldsymbol{P}$, Composite confocal images of embryonic and wandering larval CNS show endogenous Dscam expression (revealed by immunostaining with an anti-Dscam exon 18 monoclonal Ab), following tubP-GAL4-dependent $(\boldsymbol{A}-\boldsymbol{H})$ or GAL4-C155- plus asense-GAL4-dependent (I-P) induction of various anti-Dscam RNAis, including UAS-18RNAi (E, M), UAS-18/20RNAi (B, J), UAS-19RNAi (C, $\boldsymbol{K})$, UAS-18/20RNAi plus UAS-19RNAi (D, L), UAS-22/24RNAi $(\boldsymbol{F}, \boldsymbol{N})$, UAS-23RNAi $(\boldsymbol{G}, \mathbf{0})$ or UAS-22/24RNAi plus UAS-23RNAi $(\boldsymbol{H}, \boldsymbol{P})$. Arrows point at the midline in $\boldsymbol{F}$.

Dscam was silenced by UAS-23RNAi (Fig. 2G) or UAS-22/ 24RNAi (Fig. $2 \mathrm{~F}$ ), we found that the whole Dscam distribution can be subdivided into two non-overlapping compartments that possibly exclusively contain Dscam +23 and Dscam -23 , respectively. Residual Dscam was completely restricted to the midline of the ventral ganglion, following induction of UAS-22/24RNAi (Fig. $2 F$, arrows). Closer inspection revealed the midline localization of Dscam in control embryos as well (Figs. $2 A, 3 A, D$, arrows); and the midline expression of Dscam was only abolished by induction of UAS-23RNAi (Fig. 2G) that otherwise did not affect the pattern or intensity of the endogenous Dscam expres-
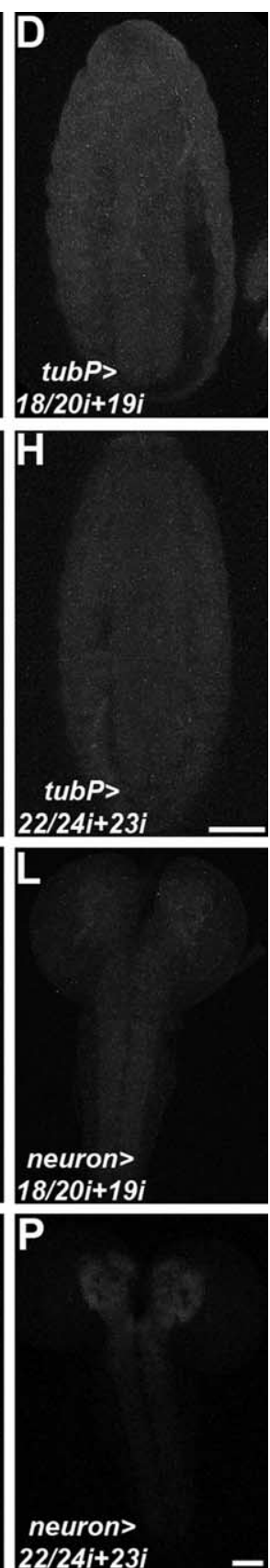

sion (Fig. $2 B, C, F$ ). These phenomena indicate that, in embryos, Dscam +19 and Dscam-19 coexist in analogous structures while Dscam +23 and Dscam -23 reside in distinct compartments.

Similar analysis revealed that most Dscams in the late larval CNS lack exon 19 and exon 23. Pan-neuronal induction of either UAS-19RNAi (Fig. $2 \mathrm{~K}$ ) or UAS23RNAi (Fig. 2O) to deplete Dscam +19 or Dscam +23 minimally affected the expression pattern or level of endogenous Dscam. In contrast to these observations, depleting Dscam-19 (Fig. 2J) or Dscam-23 (Fig. 2N) drastically reduced the Dscam expression in the late larval CNS. Notably, distinct patterns of residual Dscam were detected. Upon induction of UAS-18/20RNAi to eliminate Dscam-19 (Fig. 2J), the residual Dscam, although barely detectable, existed in a similar pattern to that of control animals (Fig. $2 I$ ). In contrast, induction of UAS-22/24RNAi to silence Dscam-23 transcripts selectively depleted endogenous Dscam from those neuropil structures where Dscam is normally enriched (Fig. 2N). These results suggest that larval development of the Drosophila CNS is mainly supported by Dscam isoforms lacking exons 19 and 23. In addition, Dscam +23 or Dscam -23 exists in distinct patterns, although not as exclusive as in embryos, and potentially governs different morphogenetic processes through development of the Drosophila CNS.

To validate some of the above conclusions, we sought to detect Dscam +19 directly by immunostaining with exon 19targeted antibody (Ab19), a polyclonal antibody raised against a peptide sequence characteristic of Dscam exon 19. The Ab19 selectively bound to ectopic Dscam+19::GFP (data not shown). Furthermore, a similar ladder-like pattern of immunocytochemical signals, which was eliminated in Dscam-null embryos, was detected following immunostaining of wild-type embryos with the Ab18 (Fig. $3 A, B$ ) or Ab19 (Fig. 3D,E). These results demonstrate that the Ab19 specifically binds with Dscam+19. When the Ab18 (Fig. 3C) or the Ab19 (Fig. 3F) were used to label all the Dscam isoforms or Dscam +19 isoforms in the late larval CNS, we detected abundant Dscam expression only with the Ab18. This substantiates the notion that Dscam +19 is largely absent during postembryonic development of the fly CNS. These results are also consistent using transgenic RNAis to silence alternative transcripts in determining the expression patterns of distinct isoforms in vivo (Fig. $2 \mathrm{~J}, \mathrm{~K}$ ).

Analysis of Dscam transcripts by RT-PCR revealed that the dynamic usage of exon 19 and exon 23 is evident even at the mRNA level (Fig. 3G). The relative abundance of Dscam tran- 


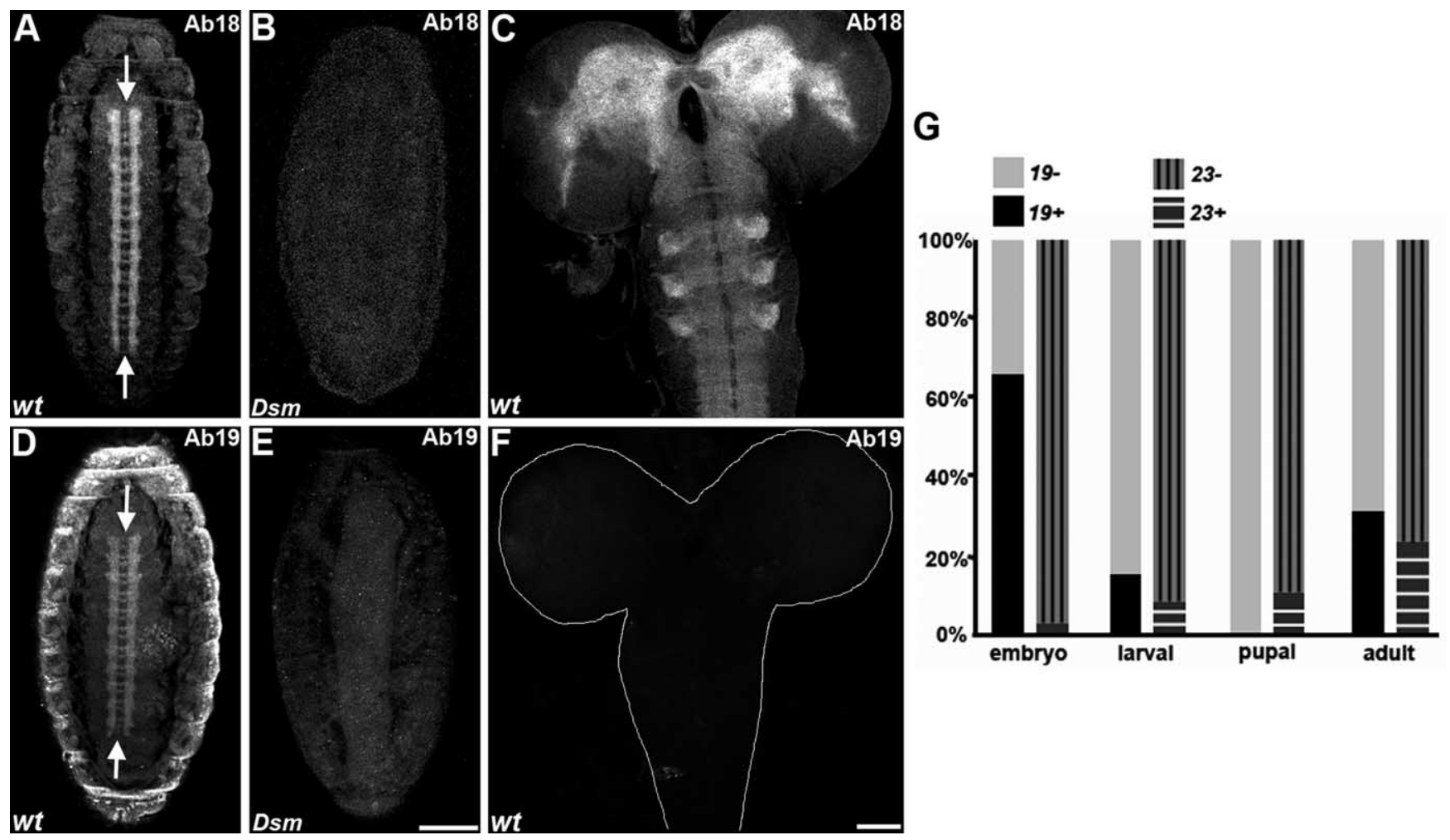

Figure 3. Stage-dependent expression of Dscam endodomain variants. $\boldsymbol{A}-\boldsymbol{F}$, An anti-Dscam exon 18 monoclonal antibody (Ab18) recognizing all Dscam proteins ( $\boldsymbol{A}-\boldsymbol{C}$ ) and an anti-Dscam exon 19 polyclonal antibody (Ab19) recognizing Dscam +19 proteins (D-F) were used to reveal the endogenous expression pattern of all Dscam variants and Dscam +19 during development. Both Ab18 and Ab19 are specific for detecting Dscam endogenous proteins as evidenced by lack of immunostaining in Dscam null mutant embryos ( $\boldsymbol{B}, \boldsymbol{E})$. Arrows point at the midline in $\boldsymbol{A}$ and $\boldsymbol{D}$. $\boldsymbol{G}, \mathrm{RT}-\mathrm{PCR}$ analysis of Dscam transcripts revealed the usage of exon 19 and exon 23. Dscam +19 transcripts exist more abundantly than Dscam - 19 transcripts at the embryonic stage, while Dscam - 19 transcripts become dominant in postembryonic CNS. In contrast, Dscam - 23 transcripts predominate over Dscam + 23 transcripts throughout development.

scripts encoding distinct endodomains varied at different developmental stages. The expression of Dscam +19 transcripts in the CNS drastically decreased from embryos (65.8\%) to larvae (15.8\%), became negligible by puparium formation $(0 \%)$, and returned after eclosion (31.6\%). In contrast, Dscam +23 transcripts rarely existed in embryos $(2.6 \%)$, and then increased in larvae $(7.9 \%)$, pupae $(10.4 \%)$ and adult brains $(21.1 \%)$. The differential expression of Dscam mRNA splicing variants at different developmental stages can account for the differences we detected in the abundance of distinct endodomains at the protein level. These results suggest that the dynamic expression of distinct Dscam endodomains is likely controlled through regulation of alternative splicing.

Together, these observations not only indicate that distinct Dscam endodomain isoforms do exist in vivo, but also demonstrate that the miRNA-based RNAi reagents work effectively to antagonize one while minimally affecting the alternative. Induction of RNAi specifically silencing one Dscam endodomain isoform (e.g., Dscam + 19 or Dscam +23) reveals the protein distribution of the other Dscam endodomain isoform (e.g., Dscam-19 or Dscam-23) in vivo. Examining the expression of Dscam +23 and Dscam -23 uncover two distinct sets of Dscampositive structures that potentially use different Dscam endodomains for supporting different morphogenetic processes.

\section{Wiring of the adult fly neural circuitry involves Dscam} transcripts lacking exons 19 and 23

Based on the above analyses of endogenous expression patterns of Dscam endodomain variants, Dscam variants lacking exons 19 and 23 may support diverse aspects of neuronal morphogenesis during larval and pupal stages. This raises the possibility that wiring of the fly neural circuitry primarily involves Dscam variants lacking exons 19 and 23. To test this hypothesis, we examined how silencing of Dscam transcripts encoding specific endodomains by targeted RNAi affects morphogenesis of two distinct types of larval-born neurons where Dscam loss-offunction phenotypes are known. We found in both MB neurons (Fig. 4A-C) and the ellipsoid body neurons (data not shown) that induction of UAS-18/20RNAi or UAS-22/24RNAi, but not UAS-19RNAi or UAS-23RNAi, effectively elicited the phenotypes characteristic of loss of Dscam function.

In MB neurons, Dscam governs axon bifurcation by preventing sister growth cones from migrating along the same path (Wang et al., 2002). It ensures formation of paired MB lobes that normally lie perpendicularly with each other and are comparable in bundle thickness (Fig. $4 \mathrm{~A}$ ). In contrast, when Dscam is absent, $\mathrm{MB}$ axons undergo excessive bifurcation and their sister branches are no longer consistently segregated. These abnormalities underlie overgrowth of one $\mathrm{MB}$ lobe at the expense of its paired one (Fig. 4B). To determine the roles of Dscam +19 , Dscam -19 , Dscam +23 or Dscam -23 in MB morphogenesis, we examined which of the above anti-Dscam miRNA transgenes could be induced to elicit these loss-of-Dscam MB phenotypes. We first demonstrated that GAL4-OK107-driven induction of UAS18RNAi caused defects in MB lobe morphology (Fig. $4 B$ ). Instead of having two comparable axon lobes, $88 \%$ of the MBs acquired one huge and one skinny lobe (Fig. 4C). This anomaly replicated Dscam mutant MB phenotypes (Wang et al., 2002). Since silencing Dscam with UAS-18RNAi eliminates all Dscam isoforms, this result has not only confirmed the efficiency of knocking down 

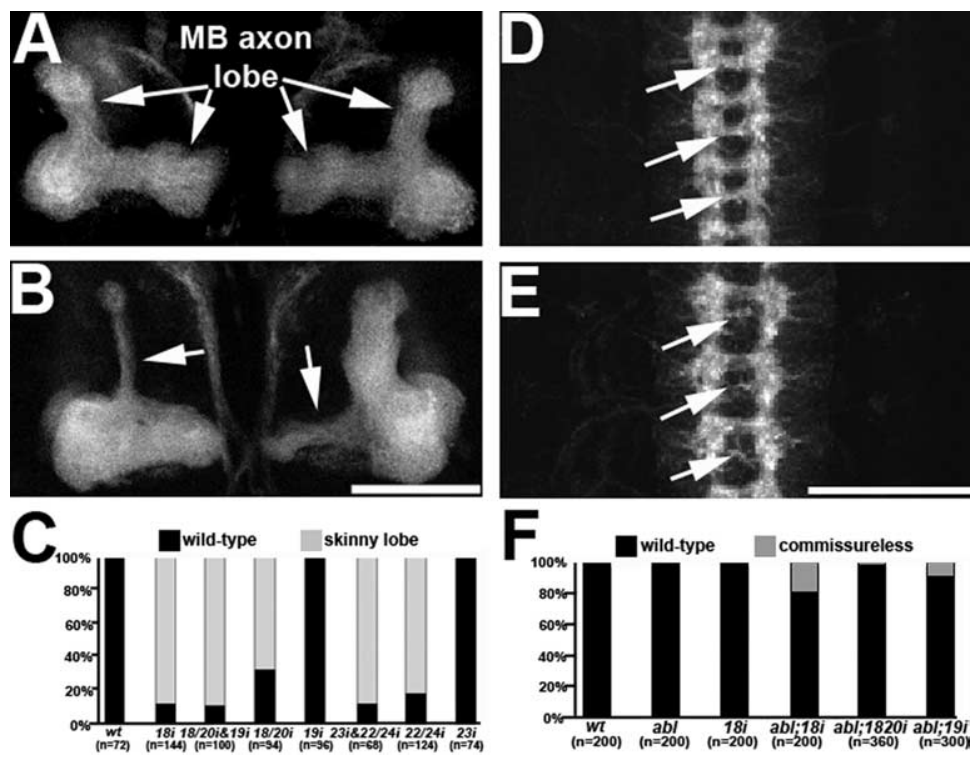

Figure 4. Role of Dscam endodomain variants in embryonic CNS and larval MB axonal morphogenesis. A-C, Quantitative analysis of wandering larval MB axon lobe ( $\boldsymbol{A}$, arrows) phenotype, following GAL4-0K107-dependent induction of various UASDscam RNAis plus UAS-mCD8::GFP. Skinny lobe phenotype (B, arrows) was observed when Dscam -19 or Dscam -23 was eliminated. D-F, Quantitative analysis of embryonic CNS commissureless phenotype, following tubP-GAL4-dependent induction of various UAS-Dscam miRNAs. No phenotype ( $\boldsymbol{D}$, arrows) was observed when all Dscam proteins were knocked down alone or in $a b l$ mutant embryos. In contrast, commissureless phenotype ( $\boldsymbol{E}$, arrows) was observed when all Dscam isoforms or Dscam +19 were eliminated in the $a b /$ mutant embryos. The embryonic CNS longitudinal fascicles and commissure pathways were visualized by immunostaining with monoclonal antibody BP102.

endogenous Dscam by transgenic RNAi, but also given us an opportunity for learning what Dscam "null" phenotypes could be obtained with similar manipulations.

We subsequently examined the effects of UAS-19RNAi, UAS18/20RNAi, UAS-23RNAi or UAS-22/24RNAi on MB morphology (Fig. 4C). While induction of UAS-19RNAi or UAS-23RNAi to deplete Dscam +19 or Dscam +23 failed to affect MB morphogenesis, 68 and $77 \%$ of the MBs acquired asymmetric axon lobes following silencing of Dscam - 19 or Dscam - 23 using UAS-18/ 20RNAi or UAS-22/24RNAi (Fig. 4C). This suggests that Dscam -19 and Dscam -23 are indispensable for gross morphogenesis of the MBs. However, the penetrance of UAS-18/ 20RNAi-induced MB phenotypes increased from 68 to $89 \%$ following coinduction with UAS-19RNAi. This implies a lesser role for Dscam +19 in mediating the neuronal morphogenetic process which is mainly governed by Dscam lacking exons 19 and 23. In contrast, coinduction of $U A S-22 / 24 R N A i$ and $U A S$ 23RNAi minimally enhanced the MB phenotype, suggesting again that Dscam +23 is dispensable to the neuronal morphogenesis. Together, these results indicate that the Dscamdependent MB lobe formation primarily involves Dscam variants lacking exons 19 and 23, which is consistent with Dscam-19-23 being predominantly expressed through postembryonic CNS development.

\section{Dscam +19 is involved in embryonic neuronal morphogenesis}

In contrast with Dscam +23 whose usage remains low through different developmental stages, both Dscam +19 and Dscam -19 are abundantly expressed in embryonic CNS. To reveal the role of Dscam exon 19 variants in neural development, we examined whether eliminating Dscam +19 or Dscam -19 affects embryonic neuronal morphogenesis. We characterized neurite tracts in the embryonic ventral ganglion that typically consists of bilater- ally symmetric longitudinal fascicles connected across the midline via repeated pairs of commissure bundles. Although aberrant neurite tracts in Dscam mutant embryos have been reported (Schmucker et al., 2000), ubiquitous induction of UAS $18 R N A i$ by tubP-GAL4 failed to disrupt the ladder-like neuronal tracts in otherwise wild-type embryos (Fig. $4 F$ ). Kidd's group recently showed that Dscam loss-offunction phenotypes in embryos can be significantly enhanced in tyrosine kinase abelson ( $a b l)$ mutant backgrounds (Andrews et al., 2008). Prompted by this observation, we examined how silencing Dscam by RNAi might affect neural tract development in $a b l$ mutant embryos (Fig. $4 D-E)$. As in the Dscam-depleted embryos caused by tubP-GAL4-dependent induction of UAS-18RNAi, the ladder-like neurite tracts were grossly normal in $a b l^{2} / a b l^{4}$ mutant embryos (Fig. $4 F$ ). In contrast, the ubiquitous induction of UAS-18RNAi in $a b l^{2} / a b l{ }^{4}$ mutant embryos effectively blocked midline crossing of neurites and caused commissureless phenotypes in $19 \%$ of the ventral ganglion segments (Fig. $4 E, F)$. These observations set the stage for determining the roles of various Dscams in embryonic neuronal morphogenesis by RNAi using distinct Dscam-targeted miRNAs.

To tell the respective contributions made by Dscam +19 and Dscam - 19, we silenced Dscam transcripts containing or lacking exon 19 in the $a b l^{2} / a b l^{4}$ mutant background. Following analogous induction with tubP-GAL4, UAS-19RNAi and UAS-18/ 20RNA $i$ elicited commissureless phenotypes in 9 and $1 \%$ of the ventral ganglion segments, respectively (Fig. $4 F$ ). Together, most embryonic Dscams carry exon 19 (Fig. 3G) and depleting exon 19-containing Dscam potently disrupts wiring of embryonic CNS. These results demonstrate that Dscam +19 plays a more important role than Dscam-19 in the development of the Drosophila embryonic CNS.

\section{Differential rescue of axon branching phenotypes by transgenic Dscam +19 or Dscam -19}

To investigate further the functions of Dscams carrying distinct endodomains, we examined whether ectopic expression of Dscams with different endodomains may act differentially in vivo. Although $\mathrm{MB}$ morphogenesis involves Dscam variants lacking exons 19 and 23, past studies have shown that the Dscam +19 +23 transgene, no matter which ectodomain it carries and whether it is fused with GFP at the C terminus or not, can effectively prevent sister branches from extending into the same axon bundle in single-cell clones of Dscam mutant neurons (Wang et al., 2004; Zhan et al., 2004; Soba et al., 2007). We wondered whether Dscam transgenes that encode Dscams with distinct endodomains may vary in their ability to restore the normal pattern of axon bifurcation and segregation in Dscam mutant MB neurons.

UAS-Dscam::GFP transgenes that encode the same ectodomain connected to one of the four possible endodomains through the exon 17.2-defined transmembrane domain were obtained. Transgenic lines with a comparable level of induc- 
tion were identified (Fig. 5A). We then examined how induction of such Dscam transgenes in Dscam mutant single-cell MARCM clones affects their axon bifurcation and segregation at the terminus of $\mathrm{MB}$ peduncle. We analyzed $\mathrm{MB} \alpha / \beta$ neurons, whose axons extend through the peduncle, undergo bifurcation at the peduncle end, and then innervate the $\mathrm{MB} \alpha$ and $\beta$ lobes with only one primary branch from each neuron (Fig. 5C). Single-cell MARCM clones of $\operatorname{MB} \alpha / \beta$ neurons were derived following mitotic recombination induced during midpupal development. Loss of the GAL4 repressor, GAL80, in the clones led to derepression of GAL4-OK107, which in turn drove expression of UAS-mCD8::GFP for marking the clones uniquely. In the rescue experiments, the derepressed GAL4OK107 also activated one of the UAS-Dscam::GFPs specifically in the clones. Given that these Dscam transgenes vary only in the presence or absence of exon 19 or 23, any differences in the phenotypes of Dscam mutant $\alpha / \beta$ neurons that have expressed different UAS-Dscam::GFPS can be fully ascribed to the differences in Dscam protein distribution and/or function due to the involvement of different endodomains.

As reported previously, single-cell MARCM clones of Dscam mutant MB $\alpha / \beta$ neurons could not effectively segregate their sister branches to prevent multiple neurites of the same cellular origin from extending into the same MB lobe (Wang et al., 2002). Although Dscam mutant neurons might acquire supernumerary branches, this segregation problem was evident even in the mutant axons that only bifurcated once at the peduncle end. Failure in the divergent segregation of sister branches appears to be the primary defect (Fig. 5D-F). Ninety-seven percent of Dscam mutant single-cell MARCM clones of $\mathrm{MB} \alpha / \beta$ neurons sent two or more neurites into one $\mathrm{MB}$ lobe, exhibiting the multibranches/lobe phenotype (Fig. 5B-F). By this measure, single-isoform Dscam-19 transgenes were comparable with single-isoform $D s c a m+19$ transgenes in the ability to correct the Dscam mutant multibranches/lobe phenotype (Fig. 5B). Failure in divergent segregation (multibranches/lobe) was observed in 22, 16, 25 , and $22 \%$ of Dscam mutant $\mathrm{MB} \alpha / \beta$ neurons (as opposed to $97 \%$ in the absence of any rescuing construct) that were rescued by the transgenes of Dscam $+19+23$, Dscam-19+23, Dscam +19-23 and Dscam-19-23, respectively (Fig. 5B). In addition, among the rescued single-cell MARCM clones (without the multibranches/lobe phenotype), we detected a single-branch/neuron phenotype in which the axon did not bifurcate at the peduncle end and stochastically projected into
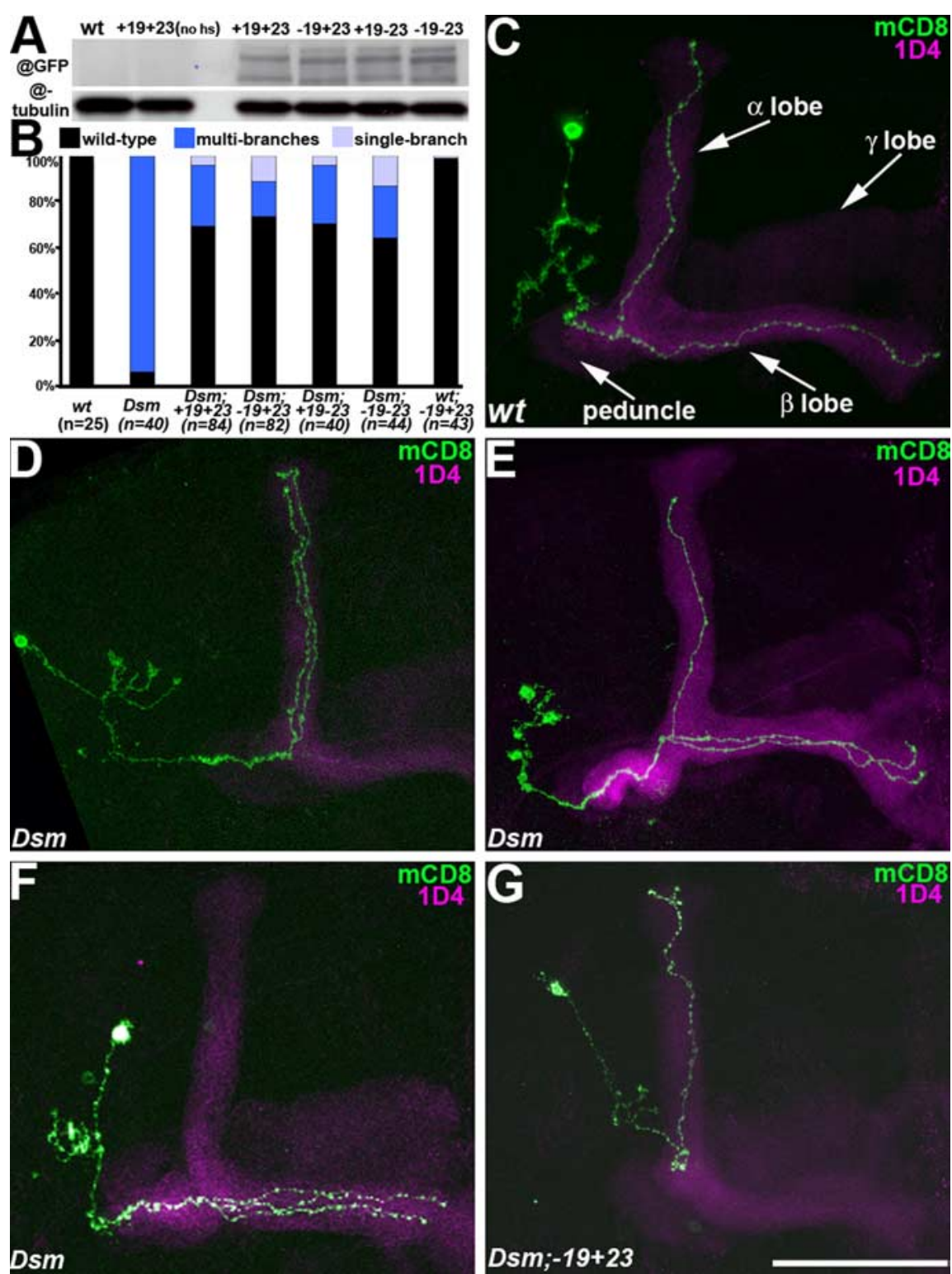

Figure 5. Rescue of $\mathrm{MB} \alpha / \beta$ multibranch phenotype by transgenic Dscam with distinct endodomains. $\boldsymbol{A}$, Dscam endodomain variants with a similar expression level were chosen to rescue the multibranch Dscam mutant MB $\alpha / \beta$ phenotype. Heat shockGAL4 was used to drive the expression of different Dscam endodomain variants by brief heat shock at $37^{\circ} \mathrm{C}$. The expression levels were determined by Western blot. $\boldsymbol{B}-\mathbf{G}$, Quantitative analysis of different $\mathrm{MB} \alpha / \beta$ neuronal phenotypes in Dscam mutant clones each of the $\mathrm{MB} \alpha / \beta$ lobes (C.D-F, In contrast, Dscam mutant single-cell clones often extend multiple branches into one MB lobe. $\mathbf{G}$, Note an unusual single-branch phenotype with no axon bifurcation at the peduncle end in some "rescued" single-cell clones. Anti-1D4 staining (magenta) reveals two strong $\alpha / \beta$ lobes and a weak $\gamma$ robe in adult MB. Anti-mCD8 antibody (green) was used to reveal mCD8::GFP in MARCM single-cell clones.

the $\alpha$ or $\beta$ lobe. Intriguingly, many more rescued single-cell MARCM clones exhibited the single-branch/neuron phenotype (no bifurcation despite presence of both $\alpha$ and $\beta$ lobes), when the mutant clones were supplemented with Dscam-19 transgenes (Fig. 5G). Rescue with Dscam-19+23 and Dscam-19-23 caused the single-branch/neuron phenotype in 11 and $13 \%$ of Dscam mutant single-cell clones (Fig. 5B). In contrast, with Dscam $+19+23$ or Dscam $+19-23$, only $5 \%$ of mutant axons failed to bifurcate at the peduncle terminus (Fig. $5 B$ ). In addition, transgenic Dscam $-19+23$ hardly affected wild-type single-cell clones; axon bifurcation at the peduncle terminus was suppressed in only 3\% of Dscam-19-23- 


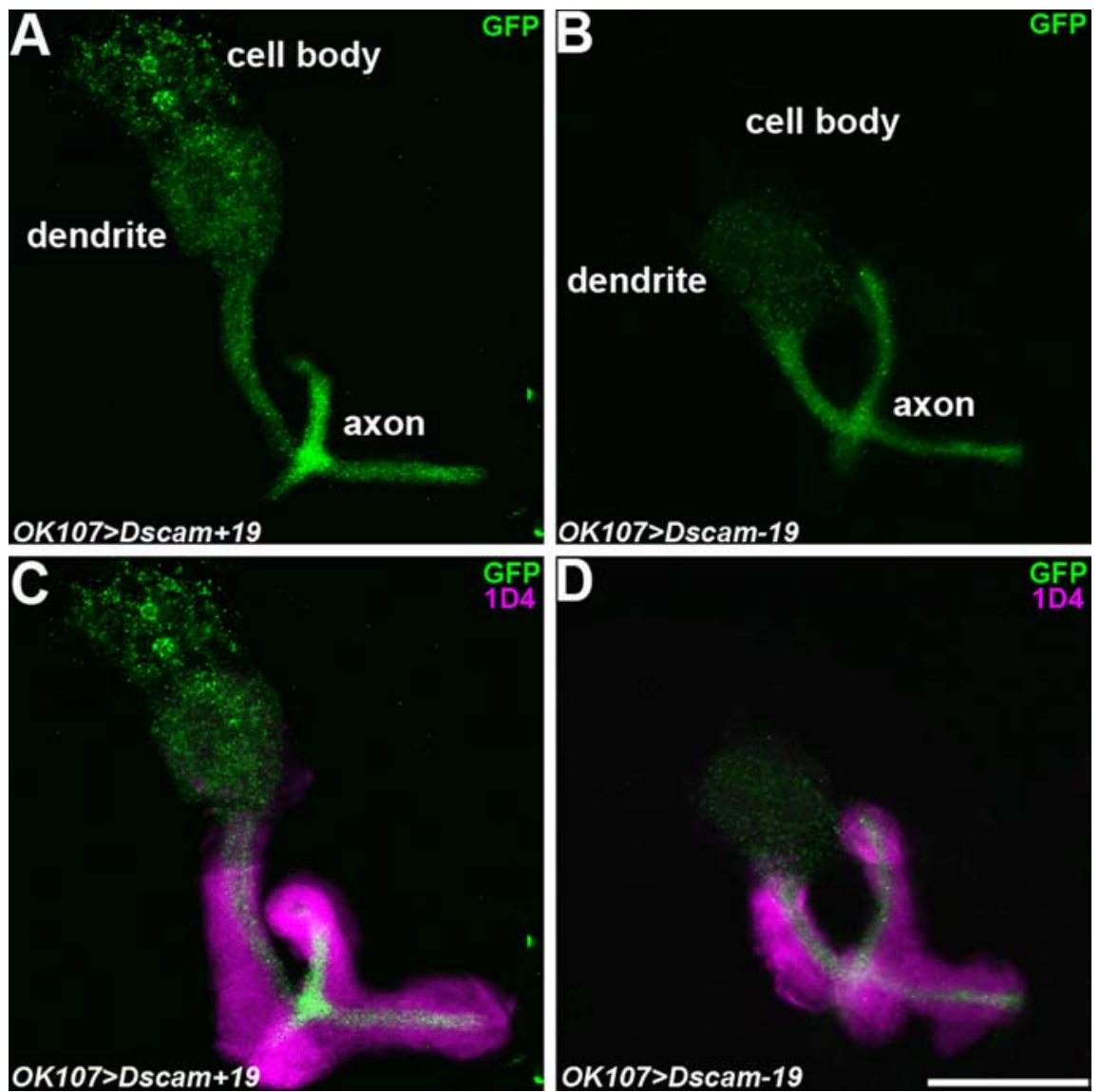

Figure 6. Exon 19-dependent differential distribution of transgenic Dscam in MB neurons. $\boldsymbol{A}-\boldsymbol{D}$, TARGET-mediated acute induction of Dscam + 19 versus Dscam -19 in larval MB neurons. Transgenic Dscam - 19::GFP was primarily targeted to the MB axon bundles and the dendritic calyx $(\boldsymbol{B}, \boldsymbol{D})$. In contrast, Dscam + 19::GFP accumulated in cell bodies as well as neurites $(\boldsymbol{A}, \boldsymbol{C})$. Antibody 1D4 (magenta) was used to reveal larval MB lobes. Anti-GFP antibody (green) was used to reveal Dscam::GFP.

expressing wild-type clones (Fig. 5B). Together, no matter whether exon 23 is present or not, Dscam-19 transgenes more potently modulate the axon bifurcation and segregation patterns than Dscam +19 transgenes. These observations suggest some exon 19-dependent modification of protein distribution or function that is relevant to where or how Dscam may signal to govern $\mathrm{MB}$ axonal morphogenesis.

\section{Differential subcellular localization of Dscam containing or lacking exon 19}

To understand the logic behind the exon 19-dependent functional differences, we visualized the protein distribution of Dscam + 19::GFP and Dscam-19::GFP transgenes in vivo. Previously, we have reported an assay to visualize Dscam protein distribution in the larval MBs by inducing UAS-Dscam::GFP transgenes in mature larval MB neurons using GAL4-201Y (Wang et al., 2004). Notably, the Dscam+19::GFP and Dscam-19::GFP were found to be enriched in cell bodies and neurites of the larval MBs, respectively (data not shown). This raises the possibility that Dscam protein distribution may vary depending on the presence or absence of exon 19.

However, GAL4-201Y drove expression of Dscam::GFPs in excessive amounts and in mature MB neurons, which is in contrast with the endogenous Dscam being expressed at a much lower level and typically in newly derived postmitotic neurons (Zhan et al., 2004). To ascertain the role of exon 19 in
Dscam protein distribution, we examined whether Dscam+19::GFP and Dscam-19::GFP remain differentially localized upon weak induction in young $\mathrm{MB}$ neurons. Transient binary induction of UAS-transgene is possible with the TARGET system, in which GAL4 activity is suppressed until inactivation of temperature-sensitive GAL80 at a restrictive temperature (McGuire et al., 2003). Derepression of GAL4-OK107 following a heat shock to inactivate GAL80 ${ }^{\text {ts }}$ allowed one to weakly express UAS-transgene in young MB neurons as judged by the central localization of neurites in the age-dependent concentrically organized axon bundles (Kurusu et al., 2002). Transient induction of UAS-Dscam::GFPs revealed that Dscam-19::GFP was selectively enriched in the neurites, while barely detectable in cell bodies (Fig. 6B,D). In contrast, Dscam + 19::GFP accumulated in cell bodies as well as neurites (Fig. $6 A, C)$. A similar pattern of differential subcellular localization of Dscam +19 and Dscam-19 was also observed when Dscam +19 and Dscam-19 transgenes were induced in projection neurons (PNs) of the antennal lobe (AL). GAL4$M z 19$ (Jefferis et al., 2004) labels a small subset of PNs that connect three $\mathrm{AL}$ glomeruli (DA1, VA1d, and DC3) to the lateral horn (LH) and MB calyx (Fig. $7 A, B)$. Upon induction with GAL4-Mz19, Dscam-19::GFPs with either transmembrane domain choice (17.1 or 17.2) were enriched in PN neurites (Fig. 7D,F). Dscam+17.1-19::GFP further accumulated in the glomeruli where PN dendrites elaborate (Fig. 7D). In contrast, Dscam+19::GFPs with either exon 17.1 or 17.2 preferentially localized in PN cell bodies (Fig. 7C,E). These results suggest that Dscam-19 is better targeted to neuronal processes, possibly explaining why transgenic Dscam-19 is more potent than the Dscam +19 in modulating postembryonic neuronal morphogenesis.

\section{Discussion}

In this study, we demonstrate that Dscam endodomain variants are dynamically and differentially expressed in the developing Drosophila CNS. This conclusion derives from: (1) the analysis of Dscam transcript compositions by RT-PCR, (2) the localization of specific Dscam endodomains by depleting the alternatives via RNAi against exon 19 , exon 23 , or the unique exon-exon junctions derived from skipping of exon 19 or exon 23 (Fig. 2), and (3) the direct visualization of Dscam +19 using Ab19 as opposed to labeling all the Dscam isoforms with Ab18 (Fig. 3). Postembryonic neuronal morphogenesis uses Dscam variants lacking exons 19 and 23 (Fig. 4C), while Dscam +19 plays a more important role in the wiring of embryonic neural tracts (Fig. $4 F$ ). Skipping exon 19 prevents accumulation of Dscams in neuronal cell bodies, implicating a mechanism for regulating Dscam protein targeting by the alternative splicing of exon 19 (Figs. 6, 7). In addition, exon 23 is dispensable for 
most Dscam-dependent neuronal morphogenetic processes but present in probably all the Dscam molecules in certain neural structures, suggesting an unidentified Dscam function in the developing Drosophila CNS (Fig. 2F).

Four different Dscam endodomain variants arise from independent alternative splicing by skipping exon 19 or exon 23. Possible approaches to study the role of these Dscam endodomain variants in neuronal morphogenesis include: (1) manipulating Dscam at the genomic level by gene targeting (Gong and Golic, 2003), or (2) silencing different Dscam endodomain transcripts by RNAi (Chen et al., 2007; Shi et al., 2007). Deleting genomic sequence to manipulate alternative exon choice might be problematic, since elimination of specific Dscam endodomain variants inevitably leads to expression of other isoforms in much broader patterns and/or at higher levels than the normal unperturbed conditions. In contrast, knock-down of Dscam endodomain variants at the translational level by RNAi should deplete the isoforms of interest while minimally affecting the expression of others. Furthermore, by targeting the junction spanning different exons (Fig. 1), the miRNA-based silencing approach allowed us to selectively deplete isoforms that lack any unique exon shared by the isoform subset. It is also worth noting that transgenic miRNA could eliminate endogenous Dscam expression at the embryonic stage, while double-strand RNA transgenes perform poorly at this stage (Fig. 2E) (H.-H. Yu and T. Lee, unpublished observations).

Using miRNA-based knockdown and immunostaining by isoform-specific antibodies, we discovered that Dscam +19 and Dscam -19 redundantly govern neuronal morphogenesis but are preferentially used at different developmental stages (Figs. 2, 3). Dscam +19 is primarily used during embryogenesis, whereas Dscam-19 abundantly exists in the postembryonic nervous system (Fig. 3D,F). Despite the dynamic changes in the relative abundance, Dscam +19 and Dscam - 19 exhibit similar spatial expression patterns (Fig. $2 B, C, J, K$ ). Consistent with these expression profiles, endogenous Dscam-19 plays an essential role in postembryonic neuronal morphogenesis (Figs. $2 \mathrm{~J}, 4 \mathrm{C}$ ), while Dscam +19 plays a role in the formation of embryonic CNS (Figs. 3D, $4 F$ ).

In contrast to the dynamic usage of exon 19, Dscam +23 and Dscam -23 are enriched in different neural structures at the same developmental stages (Fig. $2 F, G, N, O$ ). It appears that the high-expression domains exclusively consist of Dscam -23 and depleting Dscam-23 transcripts is sufficient with two circles in $\boldsymbol{C}$ and $\boldsymbol{E}$.
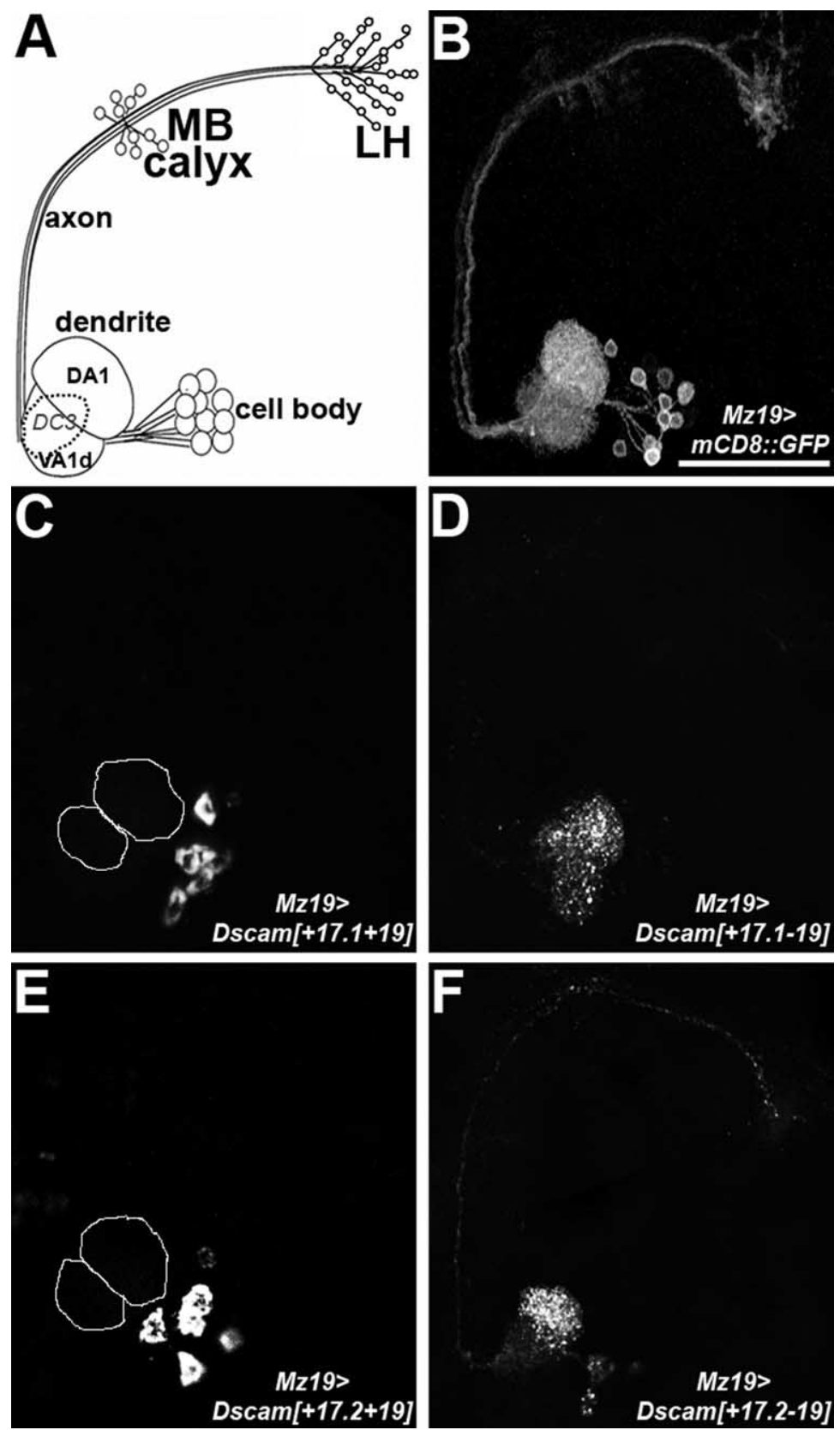

Figure 7. Differential distribution of Dscam variants in AL PNs. $\boldsymbol{A}, \boldsymbol{B}$, Schematic drawing (A) shows GAL4-Mz19-positive PNs that connect thee AL glomeruli (DA1, VA1d, and DC3) to the lateral horn (LH) ad MB calyx, as revealed by labeling of entire neuronal morphology with UAS-mCD8::GFP in $\boldsymbol{B}$. DA1 and VA1d are two prominent glomeruli and can be easily visualized in $\boldsymbol{B}, \boldsymbol{D}$, and $\boldsymbol{F}$. The position of DC3 is posterior to DA1 and VA1d and the outline of DC3 is depicted in dashed line $(\boldsymbol{A})$. $\boldsymbol{C}-\boldsymbol{F}$, Analogous induction of various UAS-Dscam::GFPs exhibits different protein distribution patterns. Transgenic Dscam + 19::GFPs primarily localize in cell bodies $(\boldsymbol{C}, \boldsymbol{E})$, while Dscam - 19::GFPs are enriched in neurites $(\boldsymbol{D}, \boldsymbol{F})$. Furthermore, as reported previously, Dscam - 19::GFP with the 17.1 transmembrane domain can be selectively targeted to dendrites (D). DA1 and VA1d are outlined

to recapitulate the loss-of-Dscam phenotype (Fig. 3C). Notably, Dscam +23 is selectively expressed in some midline cells of the embryonic CNS (Fig. 2F). Further investigation is needed to determine the identity of these midline cells (e.g., unpaired midline neurons or glia).

Skipping exon 19 produces the Dscam without a prolinerich motif and an ITAM-like (Fig. $1 A$ ). An ITAM is defined by a motif containing two tyrosine residues within the consensus sequence of $\mathrm{YxxI} / \mathrm{Lx}_{(6-12)} \mathrm{YxxI} / \mathrm{L}$ (Fodor et al., 2006). Unlike most ITAM motifs which carry Ile/Leu adjacent to the second 
tyrosine residue, AL is present in that position of Dscam (Fig. $1 A)$. The utilization of YxxA in ITAM is not unprecedented since the same variation occurs in $\mathrm{RhoH}$, a hematopoieticspecific GTPase-deficient member of Rho GTPase family (Gu et al., 2006). ITAM-mediated signals control a variety of cellular responses, ranging from phagocytosis, cell migration, proliferation, differentiation to gene induction (Fodor et al., 2006). Whether the ITAM-like in Dscam can mediate the canonical ITAM signal transduction to govern similar cellular responses awaits investigation. Intriguingly, transgenic Dscams are enriched in different subcellular compartments depending on the presence or absence of exon 19 (Fig. 6). In the MBs, transgenic Dscam-19 is preferentially targeted to neurites while transgenic Dscam +19 is distributed throughout the neurons. Induction of transgenic Dscams with different endodomains in AL PNs revealed similar patterns of differential protein distribution (Fig. 7C,F). These correlate with the phenomena that transgenic Dscam-19 acted more potently than Dscam +19 in altering neurite projection patterns in both $\mathrm{MB}$ neurons and PNs (Fig. 5) (Yu and Lee, unpublished observations). It remains to be determined whether the proline-rich motif and/or the ITAM-like within exon 19 help regulate protein distribution of Dscam.

The notable difference between Dscam +23 and Dcam -23 is the latter variant lacking exon 23 and thus losing a PDZbinding motif (Fig. 1A). However, transgenic Dscams behaved indiscriminately in the presence or absence of exon 23, yielding no insight into why Dscam-23 is used in known Dscam-dependent neuronal morphogenetic processes (Fig. $5 B)$. In addition, Dscam $+19+23::$ GFP transgene that abolishes the potential PDZ-binding motif located at the $\mathrm{C}$ terminus of Dscam remains as potent as unmodified Dscam in preventing sister branches from extending into the same axon bundle in single-cell clones of Dscam mutant neurons (Soba et al., 2007). To unravel the function of the PDZ-binding motif in Dscam +23 may require more sensitive assays or studies in different model systems.

Transgenic Dscam - 19 not only effectively prevents multiple self-branches from extending into the same MB lobe in Dscam mutant single-cell MARCM clones, but it also blocks axon bifurcation which results in single-branch/neuron phenotype in a significant number of the rescued single-cell MARCM clones (Fig. 5). The single-branch/neuron phenotype is apparently elicited by a cell-autonomous mechanism, and cannot be readily explained based on our current model about the roles of Dscam in controlling axon arborization (Fig. $5 B, G$ ). Two known mechanisms may suppress MB bifurcation at the peduncle end. First, the competition among selfbranches for the available separate fascicles probably underlies the phenomenon that neurons can reliably make the correct numbers of branches based on the numbers of fascicles that project off the branch point. This may explain why $\mathrm{MB} \alpha / \beta$ axons do not bifurcate when the $\alpha$ or $\beta$ lobe is missing (Wang et al., 2002). Second, promiscuous competition from non-self branches is thought to occur and, as a consequence, stop most axons from extending beyond the bifurcation point, when the endogenous Dscam gene loses its ectodomain diversity or a single-isoform Dscam transgene is ubiquitously expressed (Wang et al., 2004; Zhan et al., 2004; Hattori et al., 2007). Both pathological conditions are caused by environmental factors through non-autonomous mechanisms. In contrast, in the clone-specific rescue experiments, the $\alpha$ and $\beta$ lobes were both normal, and the Dscam transgene was only expressed in the neuron that exhibited the single-branch/neuron phenotype (Fig. 5G). These results clearly suggest a novel cellautonomous mechanism for Dscam in governing neurite arborization. Furthermore, it is unlikely to be due to excessive Dscam expression, since the UAS-transgenes are expressed at low levels in newborn single-cell MARCM clones, owing to the perdurance of GAL80 inherited from the heterozygous ganglion mother cells. In addition, overexpressing UASDscam $-19+23$ in wild-type MB $\alpha / \beta$ MARCM clones should increase the overall Dscam amount, but did not cause obvious single-branch/neuron phenotype (Fig. 5B). Therefore, the suppression of axon bifurcation is likely to result from loss of Dscam ectodomain diversity in a single neuron. This suggests that the huge molecular diversity in the Dscam ectodomain is not only essential for self-recognition among numerous migrating growth cones but also critical to the behavior of an isolated growth cone. It is possible that the complexity of the expressed Dscam ectodomains in a given neuron at a given time may determine the strength of Dscam-Dscam homophilic signaling between the nascent split growth cones and, thus, help govern how divergently the sister growth cones should migrate away without compromising each other.

Together, we substantiate the presence of four possible Dscam endodomains and demonstrate that Dscams with specific endodomains support specific neural developmental processes. The identification and characterization of Dscam endodomains are essential for further elucidation of the roles of Dscam and its immense molecular diversity in neural development as well as the innate immunity of insects (Watson et al., 2005). It also shed new light on how the diversity in the Dscam ectodomain may cell-autonomously govern neurite arborization in the development of Drosophila CNS.

\section{References}

Andrews GL, Tanglao S, Farmer WT, Morin S, Brotman S, Berberoglu MA, Price H, Fernandez GC, Mastick GS, Charron F, Kidd T (2008) Dscam guides embryonic axons by Netrin-dependent and -independent functions. Development 135:3839-3848.

Brites D, McTaggart S, Morris K, Anderson J, Thomas K, Colson I, Fabbro T, Little TJ, Ebert D, Du Pasquier L (2008) The Dscam homologue of the crustacean Daphnia is diversified by alternative splicing like in insects. Mol Biol Evol 25:1429-1439.

Chen BE, Kondo M, Garnier A, Watson FL, Püettmann-Holgado R, Lamar DR, Schmucker D (2006) The molecular diversity of Dscam is functionally required for neuronal wiring specificity in Drosophila. Cell 125:607-620.

Chen CH, Huang H, Ward CM, Su JT, Schaeffer LV, Guo M, Hay BA (2007) A synthetic maternal-effect selfish genetic element drives population replacement in Drosophila. Science 316:597-600.

Fodor S, Jakus Z, Mócsai A (2006) ITAM-based signaling beyond the adaptive immune response. Immunol Lett 104:29-37.

Gong WJ, Golic KG (2003) Ends-out, or replacement, gene targeting in Drosophila. Proc Natl Acad Sci U S A 100:2556-2561.

Gu Y, Chae HD, Siefring JE, Jasti AC, Hildeman DA, Williams DA (2006) RhoH GTPase recruits and activates Zap70 required for T cell receptor signaling and thymocyte development. Nat Immunol 7:1182-1190.

Jefferis GS, Vyas RM, Berdnik D, Ramaekers A, Stocker RF, Tanaka NK, Ito K, Luo L (2004) Developmental origin of wiring specificity in the olfactory system of Drosophila. Development 131:117-130.

Hattori D, Demir E, Kim HW, Viragh E, Zipursky SL, Dickson BJ (2007) Dscam diversity is essential for neuronal wiring and self-recognition. Nature 449:223-227.

Kurusu M, Awasaki T, Masuda-Nakagawa LM, Kawauchi H, Ito K, Furukubo-Tokunaga K (2002) Embryonic and larval development of the Drosophila mushroom bodies: concentric layer subdivisions and the role of fasciclin II. Development 129:409-419.

Lee T, Luo L (1999) Mosaic analysis with a repressible cell marker for studies of gene function in neuronal morphogenesis. Neuron 22:451-461. 
Matthews BJ, Kim ME, Flanagan JJ, Hattori D, Clemens JC, Zipursky SL, Grueber WB (2007) Dendrite self-avoidance is controlled by Dscam. Cell 129:593-604.

McGuire SE, Le PT, Osborn AJ, Matsumoto K, Davis RL (2003) Spatiotemporal rescue of memory dysfunction in Drosophila. Science 302:1765-1768.

Schmucker D, Clemens JC, Shu H, Worby CA, Xiao J, Muda M, Dixon JE, Zipursky SL (2000) Drosophila Dscam is an axon guidance receptor exhibiting extraordinary molecular diversity. Cell 101:671-684.

Shi L, Yu HH, Yang JS, Lee T (2007) Specific Drosophila Dscam juxtamembrane variants control dendritic elaboration and axonal arborization. J Neurosci 27:6723-6728.

Soba P, Zhu S, Emoto K, Younger S, Yang SJ, Yu HH, Lee T, Jan LY, Jan YN (2007) Drosophila sensory neurons require Dscam for dendritic selfavoidance and proper dendritic field organization. Neuron 54:403-416.

Wang J, Zugates CT, Liang IH, Lee CH, Lee T (2002) Drosophila Dscam is required for divergent segregation of sister branches and suppresses ectopic bifurcation of axons. Neuron 33:559-571.
Wang J, Ma X, Yang JS, Zheng X, Zugates CT, Lee CH, Lee T (2004) Transmembrane/juxtamembrane domain-dependent Dscam distribution and function during mushroom body neuronal morphogenesis. Neuron 43:663-672.

Watson FL, Püttmann-Holgado R, Thomas F, Lamar DL, Hughes M, Kondo M, Rebel VI, Schmucker D (2005) Extensive diversity of Ig-superfamily proteins in the immune system of insects. Science 309:1874-1878.

Wojtowicz WM, Flanagan JJ, Millard SS, Zipursky SL, Clemens JC (2004) Alternative splicing of Drosophila Dscam generates axon guidance receptors that exhibit isoform-specific homophilic binding. Cell 118:619-633.

Zhan XL, Clemens JC, Neves G, Hattori D, Flanagan JJ, Hummel T, Vasconcelos ML, Chess A, Zipursky SL (2004) Analysis of Dscam diversity in regulating axon guidance in Drosophila mushroom bodies. Neuron 43:673-686.

Zhu H, Hummel T, Clemens JC, Berdnik D, Zipursky SL, Luo L (2006) Dendritic patterning by Dscam and synaptic partner matching in the Drosophila antennal lobe. Nat Neurosci 9:349-355. 